OPEN ACCESS

Edited by:

Miguel Cacho Teixeira, Universidade de Lisboa, Portugal

Reviewed by:

Rohitashw Kumar,

University at Buffalo, United States Rajendra Prasad,

Jawaharlal Nehru University, India

${ }^{*}$ Correspondence:

Lan Yan

ylansmmu@sina.com

Yuan-Ying Jiang

13761571578@163.com

${ }^{\dagger}$ These authors have contributed equally to this work.

Specialty section:

This article was submitted to Antimicrobials, Resistance

and Chemotherapy,

a section of the journal

Frontiers in Microbiology

Received: 20 October 2017

Accepted: 30 January 2018

Published: 20 February 2018

Citation

Lv Q-Z, Qin Y-L, Yan L, Wang L, Zhang $C$ and Jiang Y-Y (2018) NSG2 (ORF19.273) Encoding Protein Controls Sensitivity of Candida albicans to Azoles through Regulating the Synthesis of C14-Methylated Sterols. Front. Microbiol. 9:218. doi: 10.3389/fmicb.2018.00218

\section{NSG2 (ORF19.273) Encoding Protein Controls Sensitivity of Candida albicans to Azoles through Regulating the Synthesis of C14-Methylated Sterols}

\author{
Quan-Zhen Lv'1t, Yu-Lin Qin ${ }^{1+}$, Lan Yan ${ }^{1 *}$, Liang Wang ${ }^{1}$, Chuyue Zhang ${ }^{2}$ and \\ Yuan-Ying Jiang ${ }^{1 *}$ \\ ${ }^{1}$ Center for New Drug Research, College of Pharmacy, Second Military Medical University, Shanghai, China, \\ ${ }^{2}$ Shanghai Pinghe Bilingual School, Shanghai, China
}

Antifungal azole drugs inhibit the synthesis of ergosterol and cause the accumulation of sterols containing a $14 \alpha$-methyl group, which is related to the properties of cell membrane. Due to the frequent recurrence of fungal infections and clinical longterm prophylaxis, azole resistance is increasing rapidly. In our research, Nsg2p, encoded by the ORF19.273 in Candida albicans, is found to be involved in the inhibition of $14 \alpha$-methylated sterols and resistance to azoles. Under the action of fluconazole, $n s g 2 \Delta / \Delta$ mutants are seriously damaged in the integrity and functions of cell membranes with a decrease of ergosterol ratio and an increase of both obtusifoliol and $14 \alpha$-methylfecosterol ratio. The balance between ergosterol and $14 \alpha$-methyl sterols mediated by NSG2 plays an important role in C. albicans responding to azoles in vitro as well as in vivo. These phenotypes are completely different from those of Nsg2p in Saccharomyces cerevisiae, which is proved to increase the stability of HMG-CoA and resistance to lovastatin. Based on the evidence above, it is indicated that the decrease of $14 \alpha$-methylated sterols is an azole-resistant mechanism in C. albicans, which may provide new strategies for overcoming the problems of azole resistance.

Keywords: Candida albicans, sterols, drug resistance, azoles, NSG2

\section{INTRODUCTION}

Candida albicans is the major opportunistic pathogen that causes superficial infections as well as candidemia and deep-tissue infections in immunocompromised individuals (Kullberg and Arendrup, 2015). Approximately, $60 \%$ of all Candida infections are caused by C. albicans (Bassetti et al., 2013). Currently azoles, which target to the rate-limiting lanosterol demethylase (Erg11p) step in ergosterol biosynthesis, are the most prevalent antifungal drugs. However, azole-resistance induced by the prophylactic and prolonged treatment has become a serious problem in the clinic (Parker et al., 2014). In the Centers for Disease Control and Prevention reports published in 2013, azole-resistant Candida has been cited as a serious threat to human health. As a result, elucidating the resistance mechanisms of C. albicans and solving the problems of clinical azole-resistance has become a great challenge.

There are three classes of antifungal drugs targeted to the ergosterol synthetic pathway: allylamines, triazoles, and morpholines. Among these, triazoles have the lowest number of side effects. The antifungal mechanisms of triazoles include two aspects: one is the reduction of ergosterol and the other is the accumulation of aberrantly formed sterols with a C14 
$\alpha$-methyl group. These effects lead to damage of cell membranes (Lupetti et al., 2002; Martel et al., 2010a). In the fungal biomembrane system, ergosterol plays an indispensable role in coordinating membrane heterogeneity, preventing water penetration, and maintaining the integrity, rigidity, and fluidity of the plasma membrane (Abe et al., 2009). In both mammals and fungi, cells regulate sterol homeostasis by multiple transcriptional and post-transcriptional feedback mechanisms. In C. albicans, ergosterol biosynthesis is mainly regulated by transcription factors, such as Upc2p, Efg1p, and Ndt80p (Sellam et al., 2009; Prasad et al., 2010; Schneider and Morschhauser, 2015). Compared with the extensive research on these transcription factors, little is known about post-transcriptional regulation of sterol homeostasis in C. albicans.

In Saccharomyces cerevisiae, sterol content can be controlled by insulin-induced genes (INSIGs) interacting directly with proteins containing sterol-sensing domains (SSDs), which is an important post-transcriptional regulation (Espenshade and Hughes, 2007). The Nsg1p and Nsg2p, classified to INSIGs in S. cerevisiae, control the sterol-dependent degradation of HMGCoA reductase. Nsg1p and Nsg2p increase the stabilization of Hmg2p by a direct interaction with the SSDs and decrease the endoplasmic reticulum (ER)-associated degradation (ERAD) of HMG-CoA reductase. This process is dependent on the lanosterol and gernanylgeranyl pyrophosphate (GGPP) levels (Flury et al., 2005). Lanosterol promotes the association of Nsg1p and Hmg2p and improves the stability of Hmg2p. When the content of lanosterol is low and GGPP is high, the physical association between Hmg2p and Nsg1p is compromised. Both Hmg2p and Nsg1p then undergo ubiquitination and degradation by ERAD pathways (Theesfeld and Hampton, 2013). NSG2 was considered to have a similar function to NSG1. The NSG1/NSG2 double deficient $S$. cerevisiae is hypersensitive to lovastatin, which reduces cholesterol levels through a competitive inhibition of HMG-CoA reductase in mammals.

We investigated whether there are INSIG genes regulating the ergosterol biosynthesis in C. albicans. Through similarity comparisons, we identified that C. albicans C3_02820C_A (ORF19.273), containing an INSIG domain, is orthologous to NSG2 in S. cerevisiae and therefore we designated it as NSG2. We created a null mutant of NSG2 in C. albicans; in contrast to the functions of NSG1/NSG2 in S. cerevisiae, an NSG2-deficient C. albicans strain improved the sensitivity to azoles and showed no difference to other stresses compared with its parental strain SN152. Hypersensitivity of $n s g 2 \Delta / \Delta$ to azoles was caused by the imbalance of sterols, especially the increase of eburicol and obtusifoliol. Our study gives a new insight in understanding the synthesis of sterols in C. albicans and the roles of sterol homoeostasis in resistance to azole stress.

\section{MATERIALS AND METHODS}

\section{Media and Agents}

Unless otherwise specified, all the strains were grown in yeast peptone dextrose (YPD, 2\% dextrose, $2 \%$ peptone, $1 \%$ yeast extract) at $30^{\circ} \mathrm{C}$. Synthetic complete (SC) medium (2\% dextrose,
$0.67 \%$ yeast nitrogen base, and amino acid mixture) was supplemented with histidine, leucine, or arginine. Fluconazole, ketoconazole, miconazole, lovastatin, terbinafine were purchased from Sigma. DMSO, ether, and cyclohexane were purchased from Sinopharm Chemical Reagent, Co., Ltd.

\section{Strain Construction}

The C. albicans strains used in this study are listed in Supplementary Table S1 and all the oligonucleotides used are listed in Supplementary Table S2. In summary, to generate the null $C$. albicans NSG2 mutant, the two alleles were sequentially disrupted with the C. maltosa LEU2 and C. dubliniensis ARG4 markers. A complementing NSG2 gene was introduced on a DNA fragment containing the C. dubliniensis HIS1 marker (Noble and Johnson, 2005). The auxotrophic markers were amplified from plasmid pSN69, pSN52, and pSN40 using the primers of LY232 and LY233 (Schaub et al., 2006). In order to generate the homologous complementing, we designed P1 and P3 primers to amplify the 289 bp cassette at 117 bp upstream of NSG2. Primers P4 and P6 were used to amplify the 201 bp cassette at 123 bp downstream of NSG2. Fusion PCR was used to amplify the disruption cassette of NSG2 (NSG2 $\Delta::$ C.m.Leu2 and NSG2 $\Delta:: C . d . A R G 4$ disruption cassette). These cassettes were transformed into SN152 using the LiAC protocol. The correct NSG2 disruptions were confirmed by PCR using the identification primers. The same strategy was used to construct the NSG2 reintroduction strains and the oligonucleotides used are listed in Supplementary Table S2. The reintroductions were identified by selection for the HIS1 auxotrophic marker.

\section{Drug Susceptibility Testing}

MIC $_{80}$ was determined by growth in RPMI1640 medium for $24 \mathrm{~h}$ as mentioned in CLSI M27-A (Raiesi et al., 2017). Briefly, C. albicans was cultured in YPD for $16 \mathrm{~h}$, and washed with PBS for three times. The fungal suspension was adjusted to $5 \times 10^{3}$ CFU/ml with RPMI1640 medium. $100 \mu \mathrm{l}$ of such inoculum was added into the 96 well plates, except for the first well which was the medium control. Drugs were added to the second well and diluted twofold serially. After cultured in $30^{\circ} \mathrm{C}$ for $24 \mathrm{~h}, \mathrm{OD}_{630}$ was measured by microplate reader. The lowest concentration of antifungal drug that was sufficient to inhibit $80 \%$ of fungal growth was designated as MIC80.

Sensitivities of strains to different drugs were further examined on the YPD agar plates by spot assay. Drugs were dissolved in DMSO as a stock solution and added to the prewarmed YPD media containing 2\% agar. C. albicans were cultured in YPD for $16 \mathrm{~h}$ and adjusted to a density of $\mathrm{OD}_{600}=0.40$ in PBS buffer. Five suspensions of fivefold serial dilution of $C$. albicans were spotted to plates containing drugs at the indicated concentrations in a volume of $3 \mu \mathrm{l}$. Finally, the plates were incubated at $30^{\circ} \mathrm{C}$ for 2 days.

\section{Transmission Electron Microscopy}

The membrane changes caused by the disruption of NSG2 or by fluconazole were imaged by transmission electron microscopy. Briefly, $500 \mu \mathrm{l}$ of $C$. albicans cultured in YPD medium overnight 
were added to $50 \mathrm{ml}$ fresh YPD medium with or without indicated concentrations of fluconazole and incubated at $30^{\circ} \mathrm{C}$ for $8 \mathrm{~h}$ with shaking at $200 \mathrm{rpm}$. After centrifugation, cell pellets were fixed with $2.5 \%$ glutaraldehyde in $0.1 \mathrm{M}$ cacodylate buffer at $4^{\circ} \mathrm{C}$ for $24 \mathrm{~h}$. Post-fixation was carried out in $1 \%$ osmium tetroxide in cacodylate buffer. After that, the pellets were dehydrated in acetone and embedded in epon. Ultra thin sections were stained with $12.5 \%$ alcoholic uranyl acetate and viewed using a HITACHI H-7650 transmission electron microscope at $80 \mathrm{kV}$. Ultrastructure of SN152 and NSG2 null mutant were compared to assess the effects of compounds.

\section{Determination of Cell Membrane Permeability by PI Staining}

The concentration of $C$. albicans was adjusted to $5 \times 10^{6} \mathrm{CFU} / \mathrm{ml}$ with RPMI1640 medium containing different concentrations of fluconazole. A total of $1.5 \mathrm{ml}$ RPMI1640 medium with C. albicans were transferred to different tubes and shaken at $200 \mathrm{rpm}$ for another $8 \mathrm{~h}$. Cells were centrifuged to remove the RPMI1640 medium and washed three times with PBS. $1 \times 10^{6}$ cells were stained by $2 \mu \mathrm{M}$ propidium iodide (PI) for $50 \mathrm{~min}$ at $30^{\circ} \mathrm{C}$. After staining, cells were washed three times with PBS and fixed with $4 \%$ paraformaldehyde. BD flow cytometry was used to detect the percentage of PI positive cells (Novickij et al., 2017).

\section{Quantitation of the Percentage of Ergosterol}

The method of Arthington-Skaggs et al. (1999) with slight modifications was used to determine the effect of NSG2 on ergosterol biosynthesis in C. albicans. Total intracellular ergosterol was quantified in the presence and absence of fluconazole in C. albicans. Briefly, $100 \mu \mathrm{l}$ of different strains were inoculated into $10 \mathrm{ml}$ of YPD with or without the indicated drugs and incubated at $30^{\circ} \mathrm{C}$ for $8 \mathrm{~h}$ at $200 \mathrm{rpm}$. Cells were washed twice with $\mathrm{ddH}_{2} \mathrm{O}$, and the weight of wet cell pellet was adjusted to $0.7 \mathrm{~g}$. Further, $3 \mathrm{ml}$ of $15 \% \mathrm{NaOH}$ resolved in $90 \%$ ethanol was added to each pellet and mixed thoroughly. The suspension was incubated at $90^{\circ} \mathrm{C}$ for $1 \mathrm{~h}$ in water bath. Sterols were extracted by addition of $1 \mathrm{ml}$ of distilled water and $3 \mathrm{ml}$ of $n$-heptane. The mixture was vortexed vigorously for $5 \mathrm{~min}$ and allowed to stand for $15 \mathrm{~min}$. The heptane layer was transferred to clear glass tubes and stored at $-20^{\circ} \mathrm{C}$. Prior to analysis $1 \mathrm{ml}$ of each sterol aliquot was diluted fivefold in 100\% ethanol and scanned between 230 and $300 \mathrm{~nm}$ using UVvisible spectrophotometer. A similar dilution of heptane in $100 \%$ ethanol was used as the blank. $50 \mu \mathrm{g}$ of cholesterol was added to each sample as the internal reference for the quantification of other sterols. The silylation of sterols is derivatized with TMSI in $30 \mathrm{ml}$ pyridine for $1 \mathrm{~h}$ at $60^{\circ} \mathrm{C}$. A $2 \mu \mathrm{l}$ aliquot was injected into the gas chromatograph-mass spectrometry (Finnigan Voyager, United States) with HP-50 columns (50\% Phenyl-50\% methylpolysiloxane, $30 \mathrm{~m} \times 0.25 \mathrm{~mm} \times 0.25 \mu \mathrm{m})$. Sterols of interest were identified by their relative retention times and mass spectra compared with the sterol profiles of NIST.

\section{Systemic Murine Candidiasis Model}

Groups of BALB/C female mice (6-8 weeks) were infected with different strains via lateral tail vein with $200 \mu \mathrm{l}$ saline containing $5 \times 10^{5} \mathrm{CFU}$ C. albicans. Fluconazole was administered to the infected mice at a dose of $2 \mathrm{mg} / \mathrm{kg}$ once a day intraperitoneally for a week starting $2 \mathrm{~h}$ after the injection with C. albicans. Mice were monitored daily for survival for a period of 50 days. KaplanMeier analyses were used to indicate the survival probabilities and Log-rank testing was used to evaluate the significance of survival curves.

\section{RESULTS}

\section{The Deficiency of NSG2 Specifically Increased Sensitivity of C. albicans to Azoles}

In S. cerevisiae, the disruption of both NSG1 and NSG2 leads to an increased sensitivity to lovastatin, which inhibits the synthesis of mevalonate. In our study, C. albicans NSG2 disrupted strains were constructed (Supplementary Figure S1). The growth rate and the formation of hyphae or biofilm were investigated and we found that NSG2 disruption had no effect on the proliferation and morphological transformation of C. albicans (Supplementary Figure S2). Unlike S. cerevisiae, in C. albicans lovastatin $(8 \mu \mathrm{g} / \mathrm{ml})$ strongly and equally inhibited the growth of both the wild-type SN152 and the $n s g 2 \Delta / \Delta$ strain (Figure 1), which suggested that NSG2 has distinct functions in $S$. cerevisiae and $C$. albicans. There are three classes of antifungal drugs targeted to the sterol synthetic pathway: allylamines (terbinafine, butenafine) target squalene epoxidase (Erg1p), triazoles (fluconazole, ketoconazole, etc.) target the lanosterol $14 \alpha$-demethylase (Erg11p) and morpholines (amorolfine, fenpropimorph) target C-8 sterol isomerase (Erg2p) and $\mathrm{C}-14$ sterol reductase (Erg24p). Antifungal susceptibility was determined by spot assays and $\mathrm{MIC}_{80}$ (Figure 1 and Table 1). The strains $n s g 2 \Delta / \Delta-1$ and $n s g 2 \Delta / \Delta-2$ were two independent transformants; they behaved almost identically. The deletion of NSG2 in C. albicans significantly enhanced the susceptibility to azoles including fluconazole, itraconazole, miconazole and ketoconazole, but reduced susceptibility to amphotericin B. However, NSG2 deletion didn't affect the susceptibility of C. albicans to terbinafine, fenpropimorph or lovastatin, which is different from lovastatin-sensitive phenotypes of $n s g 2 \Delta / \Delta$ in S. cerevisiae.

\section{The Depletion of NSG2 Disrupted the Integrity and Function of the Cell Membrane}

Ergosterol plays an important role in cell membrane integrity, permeability, and cell polarization. The ultra-structure of membranes of the SN152 and $n s g 2 \Delta / \Delta$ strains was monitored with or without the action of fluconazole via transmission electron microscopy. The deletion of NSG2 resulted in slight damage to the plasma membrane of C. albicans (Figure 2A). Moreover, in the presence of $8 \mu \mathrm{g} / \mathrm{ml}$ of fluconazole the cell 


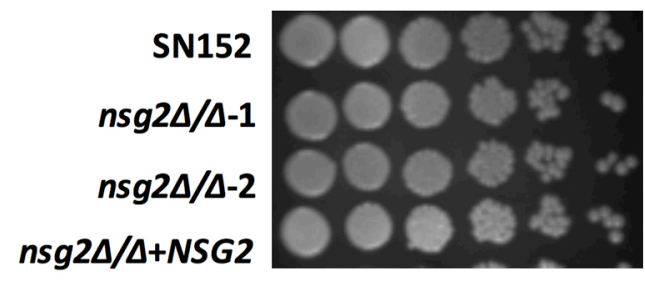

YPD

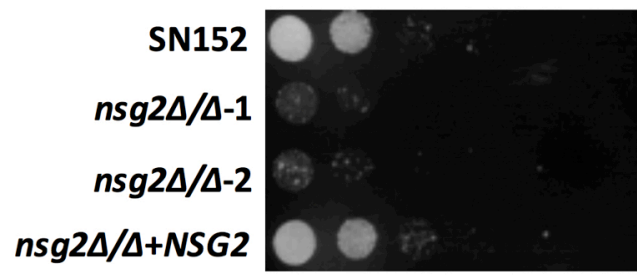

KTC

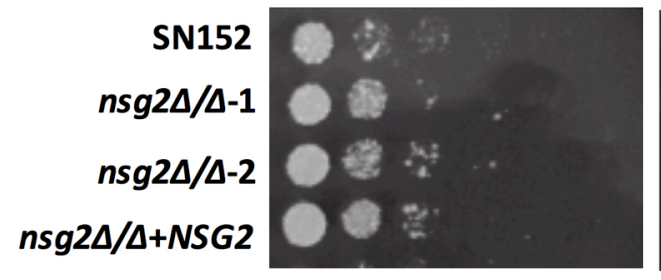

Terbinafine

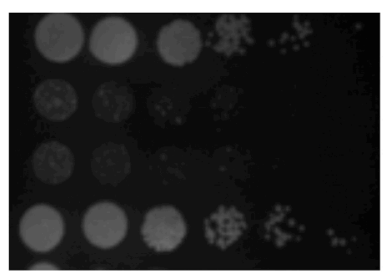

FLC

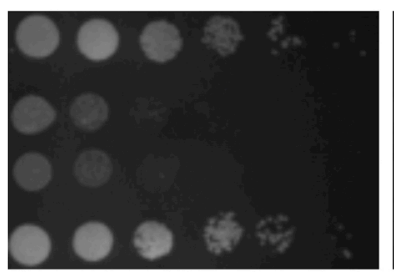

MCZ

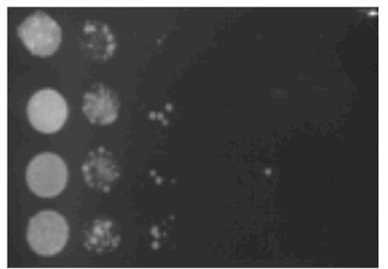

Fenpropimorpin

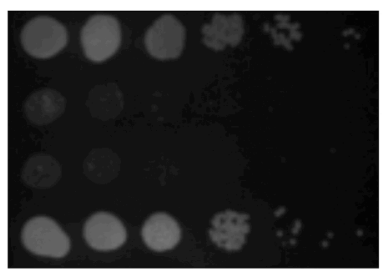

ITC

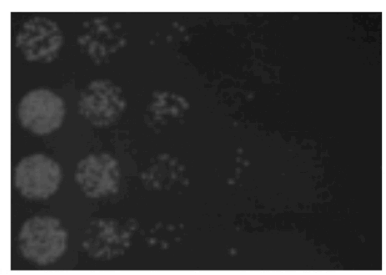

LOV

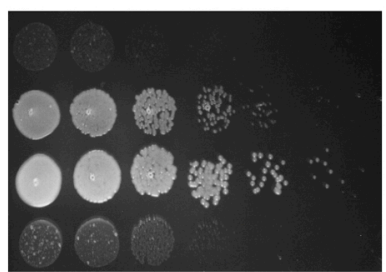

AmB

FIGURE 1 | The $n s g 2 \Delta / \Delta$ strain compromises adaptation to azoles. Spot assays comparing the sensitivity of the wild-type SN152, the NSG2-deficient and the revertant strain. Cells were grown in YPD for $16 \mathrm{~h}$, diluted, and spotted onto the plates with the indicated concentrations of different drugs. The strains were cultured on solid YPD medium and photographed after 2 days growth at $30^{\circ} \mathrm{C}$. The concentrations of each drug: fluconazole, $5 \mu \mathrm{g} / \mathrm{ml}$, itraconazole, $2.5 \mu \mathrm{g} / \mathrm{ml}$, ketoconazole, $2.5 \mu \mathrm{g} / \mathrm{ml}$, miconazole, $2.5 \mu \mathrm{g} / \mathrm{ml}$, lovastatin, $8 \mu \mathrm{g} / \mathrm{ml}$, terbinafine, $2.5 \mu \mathrm{g} / \mathrm{ml}$, fenpropimorpin, $2.5 \mu \mathrm{g} / \mathrm{ml}$, amphotericin B, $0.5 \mu \mathrm{g} / \mathrm{ml}$.

TABLE 1 | $\mathrm{MIC}_{80}(\mu \mathrm{g} / \mathrm{ml})$ values of azoles for different strains in RPMI1640 following $48 \mathrm{~h}$ incubation.

\begin{tabular}{lcccc}
\hline & SN152 & nsg2 $\mathbf{A} \mathbf{\Delta} \mathbf{- 1}$ & $\mathbf{n s g} \mathbf{\Delta} / \mathbf{\Delta - 2}$ & $\mathbf{n s g} \mathbf{2} \mathbf{\Delta} \mathbf{\Delta + N S G 2}$ \\
\hline Lovastatin & 8 & 8 & 8 & 8 \\
Fluconazole & 32 & 8 & 4 & 64 \\
Ketoconazole & 16 & 4 & 4 & 16 \\
Itraconazole & 8 & 2 & 2 & 8 \\
Miconazole & 8 & 2 & 2 & 4 \\
Terbinafine & 4 & 4 & 4 & 4 \\
\hline
\end{tabular}

membrane of strain $n s g 2 \Delta / \Delta$ shriveled dramatically and a large amount of entocyte was leaked into the gap of the cell membrane and the cell wall. Based on this observation, PI staining was used to evaluate the the effect of NSG2 deletion on cell membrane permeability (Figure 2B). In the absence of fluconazole, only a very small proportion of SN152 was stained by PI. However, in NSG2-deficient strains, approximately $2 \%$ of cells were PIpositive due to the slight cell membrane damage that we observed under the transmission electron microscopy. Consistent with this, the percentage of PI-positive cells in NSG2 disrupted strains increased significantly with the addition of 2 or $8 \mu \mathrm{g} / \mathrm{ml}$ of fluconazole (Figure 2B). These data suggested that the loss of NSG2 gene led to changes in cell membrane permeability and a slight impairment of cell membrane function. However, when we stained the yeast and cells in the early stage of hyphal formation with filipin, which combines specifically with ergosterol, we found that $n s g 2 \Delta / \Delta$ stained normally with an ergosterol-rich domain in both the yeast cells and hyphae (Supplementary Figure S3). This suggests that cell membrane damage to the $n s g 2$ $\Delta / \Delta$ strains is not caused by ergosterol loss.

\section{A Deficiency of NSG2 Increased the Accumulation of the C14-Methylated Sterols}

The azoles inhibit fungal growth due to a reduction of ergosterol and an increase in toxic sterols, which leads to decreased fluidity and permeability of the plasma membrane. Therefore, we measured the compositions of sterols in the strains by gas chromatography-mass spectrometry (GC-MS). In the absence of fluconazole, ergosterol was the major sterol in both SN152 and $n s g 2 \Delta / \Delta$. But the percentage of obtusifoliol and $14 \alpha$-methylfecosterol formed upstream of the Erg11p step were higher in $n s g 2 \Delta / \Delta$ than that in SN152 (Figures 3A,B). What's more, the obtusifoliol and $14 \alpha$-methylfecosterol accumulated in $n s g 2 \Delta / \Delta$ strains both contain a $14 \alpha$-methyl group which is fungistatic for yeast (Vale-Silva et al., 2012; Keller et al., 2015). These data suggested that NSG2 played an important role in regulating the balance of different sterols. This phenomenon was more obvious in $C$. albicans responding to fluconazole. 

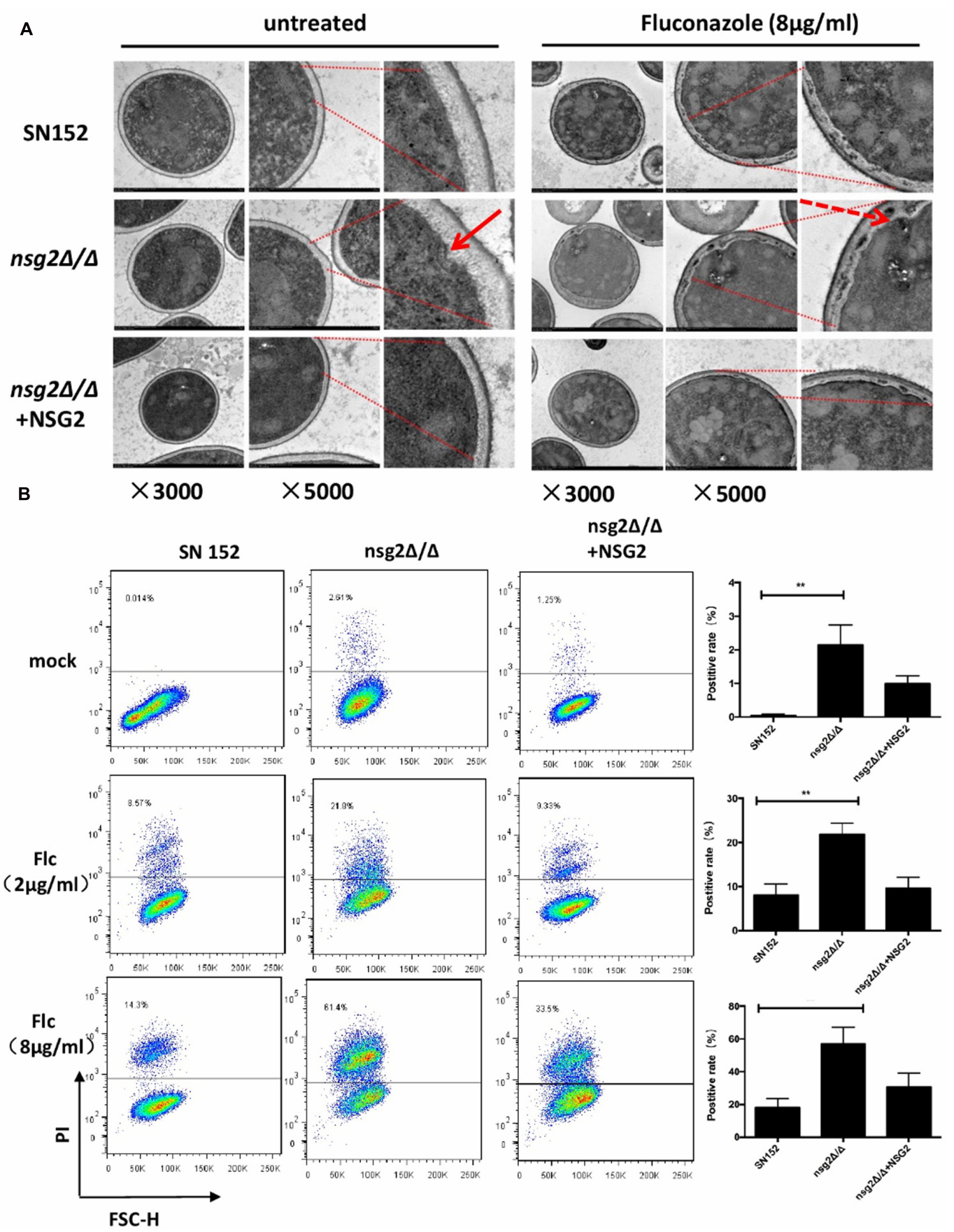

FIGURE 2 | The integrity and functions of cell membrane by NSG2 disruption. (A) Ultra-structure images of Candida albicans SN152, $n s g 2 \Delta / \Delta$ and $n s g 2 \Delta / \Delta+$ NSG2 strains in the presence or absence of $8 \mu \mathrm{g} / \mathrm{ml}$ of fluconazloe for $16 \mathrm{~h}$. Solid arrow indicated the slight damage in the membrane of $n s g 2 \Delta / \Delta$ and the dashed arrow indicated the extensive solubilization of the cytoplasmic membrane in the presence of $8 \mu \mathrm{g} / \mathrm{ml}$ of fluconazole. The magnification was indicated at the bottom of each picture. (B) Representative flow cytometric profiles and graph showing the proportions of PI ${ }^{+}-g a t e d$ cells in SN152, $n s g 2 \Delta / \Delta$ and $n s g 2 \Delta / \Delta+N S G 2$ treated with fluconazoles or not. Cells were treated with fluconazoles for $8 \mathrm{~h}$ and stained by $2 \mu \mathrm{M} \mathrm{PI}$ for 50 min. ${ }^{* *} p<0.01$ (two-tailed unpaired $t$-test).

With the treatment of $8 \mu \mathrm{g} / \mathrm{ml}$ of fluconazole, the percentage of ergosterol in both SN152 and $n s g 2 \Delta / \Delta$ decreased to about $4 \%$ and the percentage of sterols with a C14-methyl group increased. Fluconazole inhibited the activity of lanosterol $14 \alpha$-demethylase (Erg11p) and induced accumulation of lanosterol in SN152 and the reintegrate strain. However, when NSG2 was disrupted, the proportion of eburicol, obtusifoliol, and $14 \alpha$-methylfecosterol was significantly enhanced while the percentage of lanosterol in $n s g 2 \Delta / \Delta$ was lower than that in SN152 (Figure 3C). When using the squalene epoxidase inhibitor terbinafine, the difference of 

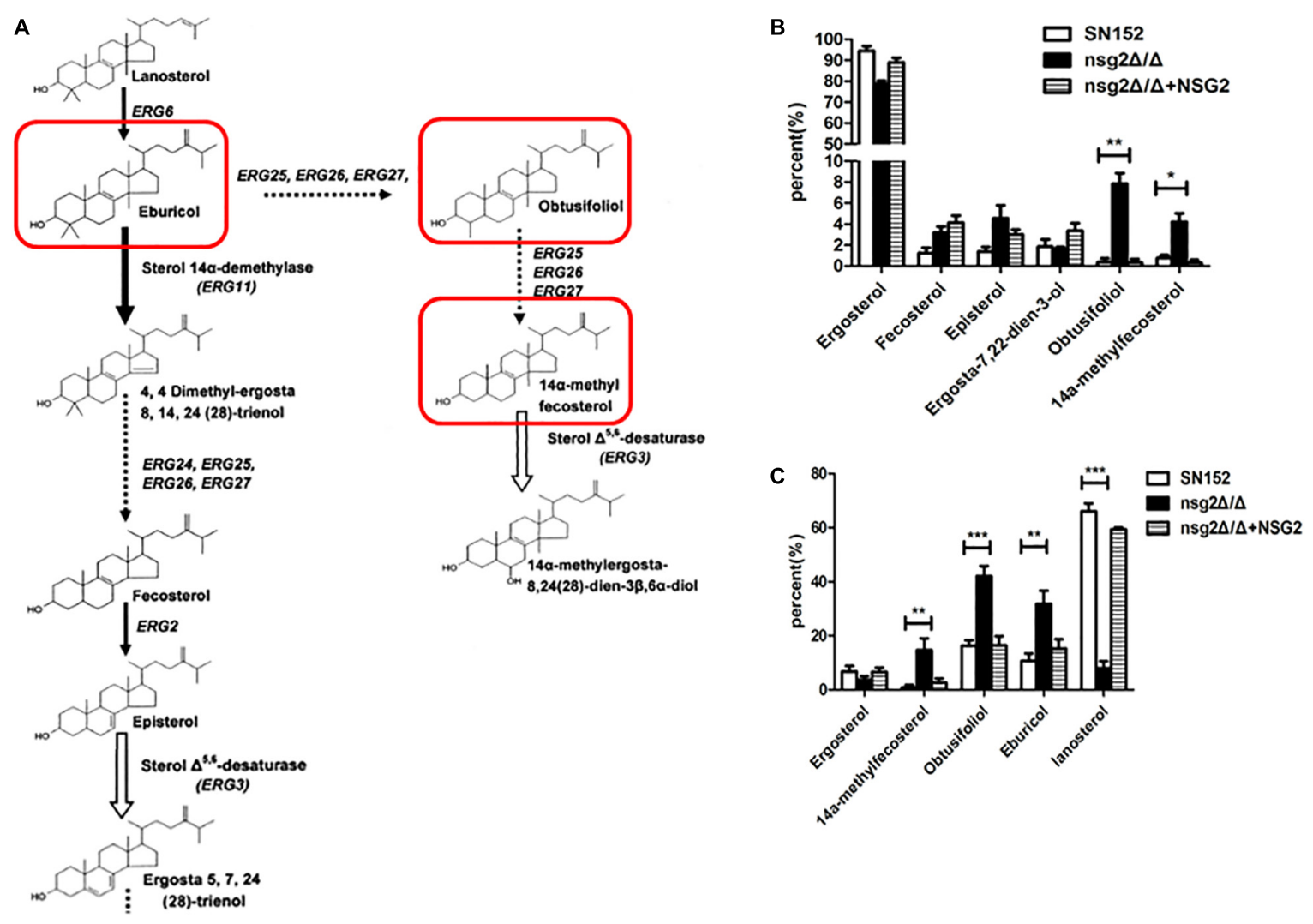

FIGURE 3 | Determination of sterols in NSG2-deficient strains by gas chromatography-mass spectrometry (GC-MS). (A) Sterol synthesis pathway in C. albicans. The red box is on behalf of accumulated sterols in $n s g 2 \Delta / \Delta$. Broken arrows, multiple enzymatic steps; solid arrows, single enzymatic step (Martel et al., 2010b). (B) Sterol compositions of SN152, $n s g 2 \Delta / \Delta$ and $n s g 2 \Delta / \Delta+$ NSG2 which were cultured in YPD medium for $16 \mathrm{~h}$ and extracted by NaOH agents. (C) Sterol compositions of SN152, nsg2 $\Delta / \Delta$ and $n s g 2 \Delta / \Delta+\mathrm{NSG} 2$ which were treated with $8 \mu \mathrm{g} / \mathrm{ml}$ of fluconazole for $6 \mathrm{~h}$. The percentage of each sterol is calculated by all detected sterols in each sample. ${ }^{*} p<0.05,{ }^{* *} p<0.01,{ }^{* * *} p<0.001$ (two-tailed unpaired $t$-test).

distribution and composition of sterols between SN152 and the NSG2 null mutant was not significant (Supplementary Figure S4). Finally, we concluded that the specific hypersensitivity of $n s g 2 \Delta / \Delta$ to azoles was attributed to the slight reduction of ergosterol and the accumulations of sterols with $14 \alpha$-methyl group. The role of NSG2 on the content of sterols may be dependent on a direct interaction between Nsg2p and Erg11p, because the transcriptional levels of ERG1, ERG7, ERG11, ERG2, and $E R G 3$ were not changed in the $n s g 2 \Delta / \Delta$ strain in comparison to those in SN152 (Supplementary Figure S5).

\section{The Disruption of NSG2 in C. albicans Increased Efficacy of Fluconazole Treatment in a Murine Model of Systemic Candidiasis}

To investigate the effects of NSG2 in maintaining the virulence of C. albicans in mice, we compared the survival rate and the renal fungal burden of strains in BALB/C mice. Without treatment with fluconazole, the survival rate and the renal fungal burden of the NSG2 null mutant were similar to those in SN152 and the reintegrate strain (Figures $\mathbf{4 A}, \mathbf{B}$ ). We then investigated whether NSG2 disruption improved the outcome of mice treated with fluconazole in vivo. The results showed that the NSG2 null mutant was almost totally cleared from the kidney. When treated with $2 \mathrm{mg} / \mathrm{kg}$ fluconazole, the survival percentage of mice infected with $n s g 2 \Delta / \Delta$ and was about $90 \%$ during 50 days (Figures 4C,D). In contrast, only $30-40 \%$ of the mice infected with SN152 or the NSG2 reintroduction strain were alive with the treatment of fluconazole after 50 days. Overall, NSG2 maintained the C. albicans resistance to azoles in vitro as well as in vivo.

\section{DISCUSSION}

The sterol biosynthesis pathway in eukaryotic cells is conserved and subject to a strict regulatory mechanism (Prasad and Singh, 2013). In C. albicans the transcriptional regulation of ERG genes includes primarily the transcriptional factors Upc2p, Efglp, and Ndt80p (Silver et al., 2004; Sellam et al., 2009; Prasad et al., 2010). Currently, the post-transcriptional regulation of the activity or stability of enzymes in the process of ergosterol synthesis is poorly studied. In mammals and yeast, the synthesis and uptake of sterol is dependent on a strict feedback control mechanism by the membrane-bound transcription factor sterol regulatory element binding protein (SREBP) (Bien and Espenshade, 2010; 

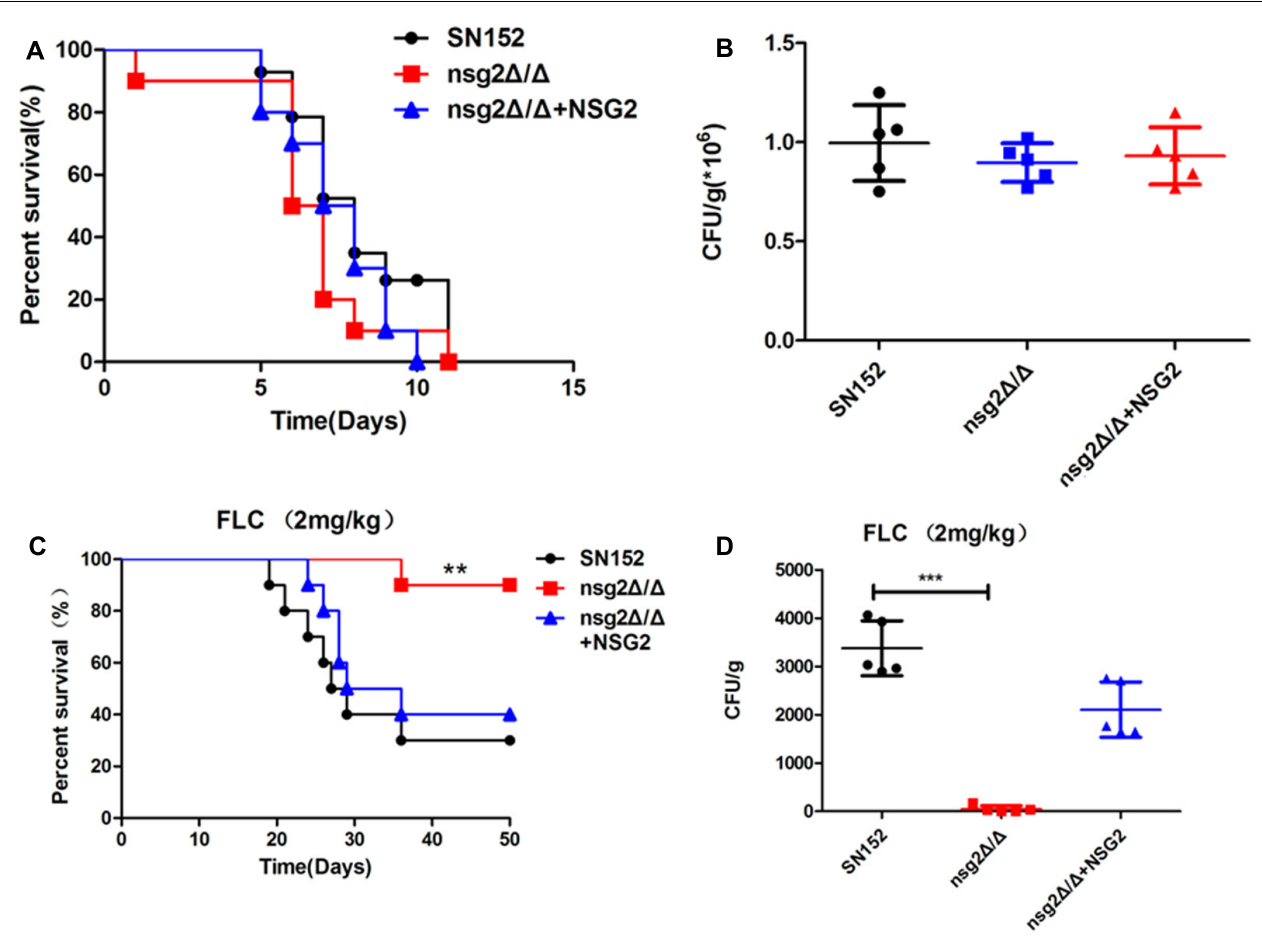

FIGURE 4 | NSG2-deficient strains were sensitive to fluconazole in vivo. (A) Survival curves of BALB/C mice infected by SN152, $n s g 2 \Delta / \Delta$ or $n s g 2 \Delta / \Delta+N S G 2$ strains. A total of $3 \times 10^{5} \mathrm{CFU}$ cells were injected to female BALB/C (6-8 weeks) through tail vein and observed until all mice dead. (B) Kidney CFU assay in mice with systemic candidiasis after infected with $3 \times 10^{5} \mathrm{CFU}$ after 2 days. (C) Survival curves of BALB/C mice infected by SN152, nsg2 $\Delta / \Delta$ or $n s g 2 \Delta / \Delta+N S G 2$ and treated with $2 \mathrm{mg} / \mathrm{kg}$ fluconazole for 1 week, ${ }^{* *} p<0.01$ (Log-rank test). (D) Kidney CFU assay in mice with systemic candidiasis after treatment with 2 mg/kg fluconazole for 7 days. ${ }^{* * *} p<0.001$ (two-tailed unpaired $t$-test).

Raychaudhuri et al., 2012). One of the best studied functions of SREBP is the regulation of HMGR transcription and steroldependent degradation, which is dependent on INSIG proteins (Espenshade and Hughes, 2007). Nsg2 in C. albicans, which is homologous to the $S$. cerevisiae INSIG protein Nsg2p, was found to be associated with resistance to azoles in our study, as the C. albicans NSG2 null mutant strain showed hypersensitivity to azoles. C. albicans cell membranes appeared to be slightly damaged in the absence of NSG2 and more seriously damaged in the presence of fluconazole.

Through the GC-MS analysis of the constitutions of sterols, we found that the hypersensitivity to azoles in $n s g 2 \Delta / \Delta$ was related to the increased eburicol, obtusifoliol, and $14 \alpha$-methylfecosterol proportions, which were almost absent in the normal strains. Eburicolis is converted to obtusifoliol and then $14 \alpha$-methylfecosterol in two successive steps under the catalyzation of $\operatorname{Erg} 25$ p, $\operatorname{Erg} 26 \mathrm{p}$ and $\operatorname{Erg} 27$, then to the toxic $14 \alpha$-methylergosta-8,24 (Maguire et al., 2014)-dien-3 $\beta, 6 \alpha$-diol under the action of Erg3p. In our research, the presence of the toxic $14 \alpha$-methylergosta-8,24 (Maguire et al., 2014)-dien$3 \beta, 6 \alpha$-diol has not been identified in $n s g 2 \Delta / \Delta$. Sanglard et al. (2003) have reported the existence of lots of obtusifoliol and a small amount of $14 \alpha$-methylfecostreol in $\operatorname{erg} 11 \Delta / \Delta$ mutants. This is very similar to the sterol distribution changes caused by NSG2 disruption in our study, although NSG2-defecient strains have a lower proportion of obtusifoliol compared with $\operatorname{erg} 11 \Delta / \Delta$. In addition, NSG2-disrupted mutants showed similar antifungal drug susceptibility compared with the heterozygous ERG11 mutants in the studies of Sanglard et al. (2003). Both of the two mutants were more sensitive to azoles and more resistant to amphotericin B compared to their wildtype strains. But there is no difference in the susceptibility to terbinafine in NSG2-disrupted mutants and the heterozygous ERG11 mutants. These changed drug sensitivities indicated that the deficiency of NSG2 may decrease the activity or stability of Erg11p and result in the changed cell membrane properties.

On the other hand, the accumulation of obtusifoliol and $14 \alpha$-fectosterol could be related to the disfunction of Erg3p which catalyzes the convention of $14 \alpha$-fectosterol to $14 \alpha$-methylergosta-8,24 (Maguire et al., 2014)-dien-3 $\beta, 6 \alpha$-diol in C. albicans treated with azoles. However, $\operatorname{erg} 3 \Delta / \Delta$ is resistant to azoles and sensitive to amphotericin $\mathrm{B}$, which is opposite to the phenotypes of the $n s g 2 \Delta / \Delta$. Consistent with this, obtusifoliol was not detected in $\operatorname{erg} 3 \Delta / \Delta$. It is speculated that NSG2-disruption may not affect the function of Erg3p on the consideration of evidence above.

The toxicity of obtusifoliol and its effect on cell membrane properties have not been fully investigated. However, there is evidence that shows the accumulation of obtusifoliol in sterols may result in an increase of C. albicans susceptibility to azoles. Specifically, Kelly et al. (1997) found higher 
obtusifoliol proportion in azole-sensitive clinical strains than that in drug-resistant strains. In previous studies, the mechanism of fluconazole resistance in $C$. albicans biofilms may be associated with the increased obtusifoliol in planktonic cells, which are sensitive to aozles (Mukherjee et al., 2003). In C. neoformans, the products formed by ERG27 inhibition, such as eburicol and obtusifolione, cannot support its growth (Mysiakina and Funtikova, 2007). Our research showed that obtusifoliol and eburicol were accumulated in $n s g 2 \Delta / \Delta$ treated with fluconazole or not, which may closely be related to its sensitivity to fluconaozle and the membrane disruption. Consistently, $n s g 2 \Delta / \Delta$ mutants were almost fully cleared from the kidneys of systemically infected mice by treatment with fluconazole in vivo. Given the critical roles of sterols in controlling the efficacy of fluconazole, Nsg2p may be a potential target for the elimination of C. albicans resistance to azoles.

Recently, Maguire et al. (2014) elaborated that the SREBPs in S. cerevisiae (Hms1) and C. albicans (Cph2) have dropped the major role in regulating sterol synthesis and instead primarily regulate filamentous growth. The regulation of sterol synthesis is replaced by the transcriptional factor Upc2p (Sellam et al., 2009). As the function of SREBPs is gradually replaced in the evolution of C. albicans, we wonder if the INSIG protein function is still conserved. Our result revealed that NSG2 containing the INSIG domain maintained the balance of ergosterol and $14 \alpha$-methylated sterols. These results mean that INSIG proteins may have lost their roles in the synthesis of ergosterol. NSG2 may affect the stability of the Erg11p, as the accumulation of eburicol, obtusifoliol, and $14 \alpha$-methylfecosterol in NSG2-deficient strains. We tested the transcriptional level of ERG11 gene and there was no difference between SN152 and the NSG2 deficient strains whether they were treated with fluconazole or not. These results suggest that Nsg2p may enhance the stability or activity of Erg $11 \mathrm{p}$ through post-translational regulations. What we have known is that the regulation of Erg11p is co-regulated with several other pathways, such as the synthesis of GPI anchors and the iron deprivation pathway (Prasad et al., 2006; Martel et al., 2010b; Hameed et al., 2011; Yadav et al., 2014). If a new protein is found to act in the

\section{REFERENCES}

Abe, F., Usui, K., and Hiraki, T. (2009). Fluconazole modulates membrane rigidity, heterogeneity, and water penetration into the plasma membrane in Saccharomyces cerevisiae. Biochemistry 48, 8494-8504. doi: 10.1021/bi9 00578y

Arthington-Skaggs, B. A., Jradi, H., Desai, T., and Morrison, C. J. (1999). Quantitation of ergosterol content: novel method for determination of fluconazole susceptibility of Candida albicans. J. Clin. Microbiol. 37, 3332-3337.

Bassetti, M., Merelli, M., Righi, E., Diaz-Martin, A., Rosello, E. M., Luzzati, R., et al. (2013). Epidemiology, species distribution, antifungal susceptibility, and outcome of candidemia across five sites in Italy and Spain. J. Clin. Microbiol. 51, 4167-4172. doi: 10.1128/JCM.01998-13

Bien, C. M., and Espenshade, P. J. (2010). Sterol regulatory element binding proteins in fungi: hypoxic transcription factors linked to pathogenesis. Eukaryot. Cell 9, 352-359. doi: 10.1128/EC.00358-09

Espenshade, P. J., and Hughes, A. L. (2007). Regulation of sterol synthesis in eukaryotes. Ann. Rev. Genet. 41, 401-427. doi: 10.1146/annurev.genet.41. 110306.130315 regulation of stability or activity of enzymes participating in the ergosterol synthesis, it may be a new target of antifungal drugs.

\section{ETHICS STATEMENT}

The research was conducted in accordance with the Declaration of Helsinki and with the Guide for Care and Use of Laboratory Animals as adopted and promulgated by the United National Institutes of Health. All mouse experimental procedures were approved by the Institutional Animal Care and Use Committee of the Second Millitary Medical University.

\section{AUTHOR CONTRIBUTIONS}

Conceived and designed the experiments: LY and Y-YJ. Performed the experiments: Q-ZL and Y-LQ. Analyzed the data: LW and CZ. Wrote the manuscript: Q-ZL, Y-LQ, and CZ.

\section{FUNDING}

This work was supported by the Shanghai Natural Science Fund (17ZR1437700) and the Natural Science Foundation of China (81330083).

\section{ACKNOWLEDGMENTS}

We thank Malcolm Whiteway (Concordia University) for critical reading of our manuscript.

\section{SUPPLEMENTARY MATERIAL}

The Supplementary Material for this article can be found online at: https://www.frontiersin.org/articles/10.3389/fmicb. 2018.00218/full\#supplementary-material

Flury, I., Garza, R., Shearer, A., Rosen, J., Cronin, S., and Hampton, R. Y. (2005). INSIG: a broadly conserved transmembrane chaperone for sterolsensing domain proteins. EMBO J. 24, 3917-3926. doi: 10.1038/sj.emboj. 7600855

Hameed, S., Dhamgaye, S., Singh, A., Goswami, S. K., and Prasad, R. (2011). Calcineurin signaling and membrane lipid homeostasis regulates iron mediated multidrug resistance mechanisms in Candida albicans. PLoS One 6:e18684. doi: 10.1371/journal.pone.0018684

Keller, P., Müller, C., Engelhardt, I., Hiller, E., Lemuth, K., Eickhoff, H., et al. (2015). An antifungal benzimidazole derivative inhibits ergosterol biosynthesis and reveals novel sterols. Antimicrob. Agents Chemother. 59, 6296-6307. doi: 10.1128/AAC.00640-15

Kelly, S. L., Lamb, D. C., Kelly, D. E., Manning, N. J., Loeffler, J., Hebart, H., et al. (1997). Resistance to fluconazole and cross-resistance to amphotericin $\mathrm{B}$ in Candida albicans from AIDS patients caused by defective sterol delta5,6-desaturation. FEBS Lett. 400, 80-82. doi: 10.1016/S0014-5793(96) 01360-9

Kullberg, B. J., and Arendrup, M. C. (2015). Invasive candidiasis. N. Engl. J. Med. 373, 1445-1456. doi: 10.1056/NEJMra1315399 
Lupetti, A., Danesi, R., Campa, M., Del Tacca, M., and Kelly, S. (2002). Molecular basis of resistance to azole antifungals. Trends Mol. Med. 8, 76-81. doi: 10.1016/ S1471-4914(02)02280-3

Maguire, S. L., Wang, C., Holland, L. M., Brunel, F., Neuvéglise, C., Nicaud, J. M., et al. (2014). Zinc finger transcription factors displaced SREBP proteins as the major Sterol regulators during Saccharomycotina evolution. PLoS Genet. 10:e1004076. doi: 10.1371/journal.pgen.1004076

Martel, C. M., Parker, J. E., Bader, O., Weig, M., Gross, U., Warrilow, A. G., et al. (2010a). A clinical isolate of Candida albicans with mutations in ERG11 (encoding sterol 14alpha-demethylase) and ERG5 (encoding C22 desaturase) is cross resistant to azoles and amphotericin B. Antimicrob. Agents Chemother. 54, 3578-3583. doi: 10.1128/AAC.00303-10

Martel, C. M., Parker, J. E., Bader, O., Weig, M., Gross, U., Warrilow, A. G., et al. (2010b). Identification and characterization of four azole-resistant erg3 mutants of Candida albicans. Antimicrob. Agents Chemother. 54, 4527-4533. doi: 10.1128/AAC.00348-10

Mukherjee, P. K., Chandra, J., Kuhn, D. M., and Ghannoum, M. A. (2003). Mechanism of fluconazole resistance in Candida albicans biofilms: phasespecific role of efflux pumps and membrane sterols. Infect. Immun. 71, 43334340. doi: 10.1128/IAI.71.8.4333-4340.2003

Mysiakina, I. S., and Funtikova, N. S. (2007). [The role of sterols in morphogenetic processes and dimorphism in fungi]. Mikrobiologiia 76, 5-18. doi: 10.1134/ S0026261707010018

Noble, S. M., and Johnson, A. D. (2005). Strains and strategies for large-scale gene deletion studies of the diploid human fungal pathogen Candida albicans. Eukaryot. Cell 4, 298-309. doi: 10.1128/EC.4.2.298-309.2005

Novickij, V., Lastauskienè, E., Švedienė, J., Grainys, A., Staigvila, G., Paškevičius, A., et al. (2017). Membrane permeabilization of pathogenic yeast in alternating sub-microsecond electromagnetic fields in combination with conventional electroporation. J. Membr. Biol. doi: 10.1007/s00232-017-9951-4 [Epub ahead of print].

Parker, J. E., Warrilow, A. G., Price, C. L., Mullins, J. G., Kelly, D. E., and Kelly, S. L. (2014). Resistance to antifungals that target CYP51. J. Chem. Biol. 7, 143-161. doi: 10.1007/s12154-014-0121-1

Prasad, R., and Singh, A. (2013). Lipids of Candida albicans and their role in multidrug resistance. Curr. Genet. 59, 243-250. doi: 10.1007/s00294-0130402-1

Prasad, T., Chandra, A., Mukhopadhyay, C. K., and Prasad, R. (2006). Unexpected link between iron and drug resistance of Candida spp.: iron depletion enhances membrane fluidity and drug diffusion, leading to drug-susceptible cells. Antimicrob. Agents Chemother. 50, 3597-3606. doi: 10.1128/AAC.00653-06

Prasad, T., Hameed, S., Manoharlal, R., Biswas, S., Mukhopadhyay, C. K., Goswami, S. K., et al. (2010). Morphogenic regulator EFG1 affects the drug susceptibilities of pathogenic Candida albicans. FEMS Yeast Res. 10, 587-596. doi: 10.1111/j. 1567-1364.2010.00639.x

Raiesi, O., Siavash, M., Mohammadi, F., Chabavizadeh, J., Mahaki, B., Maherolnaghsh, M., et al. (2017). Frequency of cutaneous fungal infections and azole resistance of the isolates in patients with diabetes mellitus. Adv. Biomed. Res. 6:71. doi: 10.4103/2277-9175.191003

Raychaudhuri, S., Young, B. P., Espenshade, P. J., and Loewen, C. Jr. (2012). Regulation of lipid metabolism: a tale of two yeasts. Curr. Opin. Cell Biol. 24, 502-508. doi: 10.1016/j.ceb.2012.05.006

Sanglard, D., Ischer, F., Parkinson, T., Falconer, D., and Bille, J. (2003). Candida albicans mutations in the ergosterol biosynthetic pathway and resistance to several antifungal agents. Antimicrob. Agents Chemother. 47, 2404-2412. doi: 10.1128/AAC.47.8.2404-2412.2003

Schaub, Y., Dunkler, A., Walther, A., and Wendland, J. (2006). New pFA-cassettes for PCR-based gene manipulation in Candida albicans. J. Basic Microbiol. 46, 416-429. doi: 10.1002/jobm.200510133

Schneider, S., and Morschhauser, J. (2015). Induction of Candida albicans drug resistance genes by hybrid zinc cluster transcription factors. Antimicrob. Agents Chemother. 59, 558-569. doi: 10.1128/AAC.04448- 14

Sellam, A., Tebbji, F., and Nantel, A. (2009). Role of Ndt80p in sterol metabolism regulation and azole resistance in Candida albicans. Eukaryot. Cell 8, 11741183. doi: 10.1128/EC.00074-09

Silver, P. M., Oliver, B. G., and White, T. C. (2004). Role of Candida albicans transcription factor Upc2p in drug resistance and sterol metabolism. Eukaryot. Cell 3, 1391-1397. doi: 10.1128/EC.3.6.1391-1397.2004

Theesfeld, C. L., and Hampton, R. Y. (2013). Insulin-induced gene protein (INSIG)-dependent sterol regulation of $\mathrm{Hmg} 2$ endoplasmic reticulumassociated degradation (ERAD) in yeast. J. Biol. Chem. 288, 8519-8530. doi: 10.1074/jbc.M112.404517

Vale-Silva, L. A., Coste, A. T., Ischer, F., Parker, J. E., Kelly, S. L., Pinto, E., et al. (2012). Azole resistance by loss of function of the sterol Delta(5),(6)desaturase gene (ERG3) in Candida albicans does not necessarily decrease virulence. Antimicrob. Agents Chemother. 56, 1960-1968. doi: 10.1128/AAC. 05720-11

Yadav, B., Bhatnagar, S., Ahmad, M. F., Jain, P., Pratyusha, V. A., Kumar, P., et al. (2014). First step of glycosylphosphatidylinositol (GPI) biosynthesis cross-talks with ergosterol biosynthesis and Ras signaling in Candida albicans. J. Biol. Chem. 289, 3365-3382. doi: 10.1074/jbc.M113. 528802

Conflict of Interest Statement: The authors declare that the research was conducted in the absence of any commercial or financial relationships that could be construed as a potential conflict of interest.

Copyright (c) 2018 Lv, Qin, Yan, Wang, Zhang and Jiang. This is an open-access article distributed under the terms of the Creative Commons Attribution License (CC BY). The use, distribution or reproduction in other forums is permitted, provided the original author(s) and the copyright owner are credited and that the original publication in this journal is cited, in accordance with accepted academic practice. No use, distribution or reproduction is permitted which does not comply with these terms. 